\title{
Erratum to: Considerations for sensitivity analysis with likelihood-based models
}

\section{Joseph W. Hogan}

Published online: 12 November 2009

(C) Sociedad de Estadística e Investigación Operativa 2009

\section{Erratum to: Test (2009) 18:59-64}

\section{DOI 10.1007/s11749-009-0142-1}

On page 60, equation (1) should read

$$
f_{\theta}\left(y_{\mathrm{obs}}, y_{\mathrm{mis}}, r\right)=f_{\theta}\left(y_{\mathrm{obs}}, r\right) f_{\theta}\left(y_{\mathrm{mis}} \mid y_{\mathrm{obs}}, r\right) .
$$

In the fourth line following equation (1), $f_{\theta}\left(y_{\text {mis }} \mid y_{\text {mis }}, r\right)$ should be replaced by $f_{\theta}\left(y_{\text {mis }} \mid y_{\mathrm{obs}}, r\right)$. Equation (2) should read

$$
f_{\theta}\left(y_{\mathrm{obs}}, y_{\mathrm{mis}}, r\right)=f_{\beta}\left(y_{\mathrm{obs}}, r\right) f_{\beta, \Delta}\left(y_{\mathrm{mis}} \mid y_{\mathrm{obs}}, r\right) .
$$

On page 61, the first line of the model described in Sect. 3.1 should read $Y_{1} \sim N\left(\mu_{1}, \sigma_{1}^{2}\right)$.

The online version of the original article can be found under doi:10.1007/s11749-009-0142-1.

J.W. Hogan ( $\triangleleft)$

Biostatistics Section, Program in Public Health, Brown University, Providence, USA

e-mail: jhogan@stat.brown.edu 